\title{
Some New Families of E-Cordial Graphs
}

\author{
S K Vaidya (Corresponding author) \\ Saurashtra University \\ Rajkot 360005, GUJARAT, India \\ E-mail: samirkvaidya@yahoo.co.in \\ Lekha Bijukumar \\ Shanker Sinh Vaghela Bapu Institute of Technology \\ Gandhinagar, GUJARAT, India \\ E-mail: dbijuin@yahoo.co.in
}

Received: May 23, 2011 Accepted: June 27, 2011 Published: November 1, 2011

doi:10.5539/jmr.v3n4p105 URL: http://dx.doi.org/10.5539/jmr.v3n4p105

\begin{abstract}
In this paper some new families E-cordial graphs are investigated. We prove that the graphs obtained by duplication of an arbitrary vertex as well as an arbitrary edge in cycle $C_{n}$ admit E-cordial labeling. In addition to this we derive that the joint sum of two copies of cycle $C_{n}$, the split graph of cycle $C_{n}$ of even order and the shadow graph of path $P_{n}$ for even $n$ are E-cordial graphs.
\end{abstract}

Keywords: E-cordial labeling, Joint sum, Shadow graphs

AMS Subject classification number(2010): $05 \mathrm{C} 78$.

\section{Introduction}

We begin with simple,finite,connected and undirected graph $G=(V(G), E(G))$ with $p$ vertices and $q$ edges. For all other standard terminology and notations we follow (Harary,F.,1972). We will provide brief summary of definitions and other information which serve as prerequisites for the present investigations.

1.1 Definition: Duplication of a vertex $v_{k}$ of a graph $G$ produces a new graph $G_{1}$ by adding a new vertex $v_{k}^{\prime}$ in such a way that $N\left(v_{k}\right)=N\left(v_{k}^{\prime}\right)$.

1.2 Definition: For a graph $G$ the split graph is obtained by duplicating its vertices altogether.

1.3 Definition: Consider a cycle $C_{n}$ and let $e_{k}=v_{k} v_{k+1}$ be an edge in it with $e_{k-1}=v_{k-1} v_{k}$ and $e_{k+1}=v_{k+1} v_{k+2}$ be its incident edges and $e_{k}^{\prime}=v_{k}^{\prime} v_{k+1}^{\prime}$ be a new edge. The duplication of an edge $e_{k}$ by an edge $e_{k}^{\prime}$ produces a new graph $G$ in such a way that $N\left(v_{k}\right) \cap N\left(v_{k}^{\prime}\right)=\left\{v_{k-1}\right\}$ and $N\left(v_{k+1}\right) \cap N\left(v_{k+1}^{\prime}\right)=\left\{v_{k+2}\right\}$.

1.4 Definition: Consider two copies of $C_{n}$, connect a vertex of the first copy to a vertex of second copy with a new edge, the new graph obtained is called the joint sum of $C_{n}$.

1.5 Definition: The shadow graph $D_{2}(G)$ of a connected graph $G$ is obtained by taking two copies of $G$ say $G^{\prime}$ and $G^{\prime \prime}$. Join each vertex $u^{\prime}$ in $G^{\prime}$ to the neighbors of the corresponding vertex $u^{\prime \prime}$ in $G^{\prime \prime}$.

1.6 Definition: If the vertices are assigned values subject to certain conditions then it is known as graph labeling.

Graph labeling have often been motivated by practical problems is one of the fascinating areas of research. A systematic study of various applications of graph labeling is carried out in (Bloom,G.S., and Golomb,S.W.,1977,p.562-570). Labeled graph plays vital role to determine optimal circuit layouts for computers and for the representation of compressed data structure. For detailed survey on graph labeling we refer to A Dynamic Survey of Graph Labeling by (Gallian,J.,2010).

1.7 Definition: A function $f$ is called graceful labeling of graph $G$ if $f: V \rightarrow\{0,1, \ldots, q\}$ is injective and the induced function $f^{*}: E \rightarrow\{1, \ldots, q\}$ defined as $f^{*}(e=u v)=|f(u)-f(v)|$ is bijective. A graph which admits graceful labeling is called a graceful graph.

Graceful labeling was introduced by (Rosa, A., 1967, p.349-355). Many illustrious works on graceful graphs provided the reason for different ways of labeling of graphs. Some variations of graceful labeling are also introduced. Some of them are edge graceful labeling, harmonious labeling, fibonacci graceful labeling, odd graceful labeling etc. 
(Cahit, I., 1987, p.201-207) introduced cordial labeling as a weaker version of graceful labeling and harmonious labeling which is defined as follows.

1.8 Definition: Let $G$ be a graph. A mapping $f: V(G) \rightarrow\{0,1\}$ is called a binary vertex labeling of $G$ and $f(v)$ is called the label of the vertex $v$ of $G$ under $f$.

For an edge $e=u v$, the induced edge labeling $f^{*}: E(G) \rightarrow\{0,1\}$ is given by $f^{*}(e=u v)=|f(u)-f(v)|$. Let $v_{f}(0), v_{f}(1)$ be the number of vertices of $G$ having labels 0 and 1 respectively under $f$ and let $e_{f}(0), e_{f}(1)$ be the number of edges of $G$ having labels 0 and 1 respectively under $f^{*}$.

1.9 Definition: A binary vertex labeling of a graph $G$ is cordial labeling if $\left|v_{f}(0)-v_{f}(1)\right| \leq 1$ and $\left|e_{f}(0)-e_{f}(1)\right| \leq 1$. A graph is cordial if it admits cordial labeling.

1.10 Definition: A graph $G=(V(G), E(G))$ with $p$ vertices and $q$ edges is said to be edge graceful if there exists a bijection $f: E(G) \longrightarrow\{1,2, \ldots, q\}$ such that the induced mapping $f^{+}: V(G) \longrightarrow\{0,1,2, \ldots, p-1\}$ given by $f^{+}(x)=$ $\left(\sum f(x y)\right)(\bmod p)$ taken over all edges $x y$ is a bijection.

1.11 Definition: Let $G=(V(G), E(G))$ with $p$ vertices and $q$ edges and $f: E(G) \rightarrow\{0,1\}$. Define $f *$ on $V(G)$ by $f(v)=\sum\{f(u v) / u v \in E(G)\}(\bmod 2)$. The function $f$ is called an $E$-cordial labeling of $G$ if the number of vertices labeled 0 and the number of vertices labeled 1 differ by at most 1 and the number of edges labeled 0 and the number of edges labeled 1 differ by at most 1 . A graph that admits E-cordial labeling is called E-cordial.

(Yilmaz, R., and Cahit, I., 1997, p.251-266) introduced E-cordial labeling which is a weaker version of edge graceful labeling having blend of cordial labeling. They proved that the trees with $n$ vertices, $K_{n}, C_{n}$ are E-cordial if and only if $n \not \equiv 2(\bmod 4)$ while $K_{m, n}$ admits E-cordial labeling if and only if $m+n \not \equiv 2(\bmod 4)$. They observed that the graphs with $p \equiv 2(\bmod 4)$ can not be E-cordial. (Devaraj, J., 2004, p.14-18) has shown that $M_{m, n}$ which is the the mirror graph of $K_{m, n}$ is E-cordial when $m+n$ is even and the generalized Petersen graph $P_{n, k}$ is E-cordial when $n$ is even.

\section{Main Results}

\subsection{Theorem: The graph obtained by duplication of an arbitrary vertex of $C_{n}$ admits E-cordial labeling.}

Proof: Let $v_{1}, v_{2}, \ldots, v_{n}$ be the vertices of the cycle $C_{n}$. Let $G$ be the graph obtained by duplicating an arbitrary vertex of $C_{n}$. Without loss of generality let this vertex be $v_{1}$ and the newly added vertex be $v_{1}^{\prime} . E(G)=\left\{E\left(C_{n}\right), e^{\prime}, e^{\prime \prime}\right\}$ where $e^{\prime}=v_{1}^{\prime} v_{2}$ and $e^{\prime \prime}=v_{n} v_{1}^{\prime}$. To define $f: E(G) \rightarrow\{0,1\}$ two cases are to be considered.

Case $1: n \equiv 0,2(\bmod 4)$

$$
\begin{aligned}
& f\left(e^{\prime}\right)=0 \\
& f\left(e^{\prime \prime}\right)=1 ; \\
& f\left(e_{i}\right)=0 ; \text { for } i \equiv 1,0(\bmod 4) \\
& =1 ; \text { for } i \equiv 2,3(\bmod 4) \text { and } 1 \leq i \leq n
\end{aligned}
$$

Case $2: n \equiv 3(\bmod 4)$

$f\left(e^{\prime}\right)=0$;

$f\left(e^{\prime \prime}\right)=1$;

$f\left(e_{i}\right)=1$; for $i \equiv 1,2(\bmod 4)$,

$=0 ;$ for $i \equiv 0,3(\bmod 4)$ and $1 \leq i \leq n$

In view of the above defined labeling pattern $f$ satisfies the conditions for E-cordial labeling as shown in Table 1. That is, the graph obtained by the duplication of an arbitrary vertex in cycle $C_{n}$ admits E-cordial labeling.

2.2 Illustration: Figure 1 shows the E-cordial labeling of the graph obtained by the duplication of an arbitrary vertex in cycle $C_{12}$.

\subsection{Theorem: The graph obtained by duplication of an arbitrary edge in $C_{n}$ admits E-cordial labeling.}

Proof: Let $v_{1}, v_{2}, \ldots, v_{n}$ be the vertices of the cycle $C_{n}$. Let $G$ be the graph obtained by duplicating an arbitrary edge of $C_{n}$. Without loss of generality let this edge be $e_{1}=v_{1} v_{2}$ and the newly added edge be $e_{1}^{\prime}=v_{1}^{\prime} v_{2}^{\prime} . E(G)=\left\{E\left(C_{n}\right), e_{1}^{\prime}, e^{\prime}, e^{\prime \prime}\right\}$ where $e^{\prime}=v_{2}^{\prime} v_{3}$ and $e^{\prime \prime}=v_{n} v_{1}^{\prime}$. Define $f: E(G) \rightarrow\{0,1\}$ as follows.

Case $1: n \equiv 1,2(\bmod 4)$

$f\left(e_{1}^{\prime}\right)=1 ;$ 
$f\left(e^{\prime}\right)=0 ;$

$f\left(e^{\prime \prime}\right)=1$;

For $1 \leq i \leq n$

$f\left(e_{i}\right)=0$; for $i \equiv 0,1(\bmod 4)$,

$$
=1 \text {; for } i \equiv 2,3(\bmod 4)
$$

Case $2: n \equiv 3(\bmod 4)$

$f\left(e_{1}^{\prime}\right)=1$;

$f\left(e^{\prime}\right)=0$;

$f\left(e^{\prime \prime}\right)=1$;

For $1 \leq i \leq n-1$

$f\left(e_{i}\right)=0$; for $i \equiv 0,1(\bmod 4)$,

$=1 ;$ for $i \equiv 2,3(\bmod 4)$

$f\left(e_{n}\right)=0 ;$

In view of the above defined labeling pattern $f$ satisfies the conditions for E-cordial labeling as shown in Table 2. That is, the graph obtained by the duplication of an arbitrary edge in cycle $C_{n}$ is E-cordial.

2.4 Illustration: The E-cordial labeling of the graph obtained by the duplication of an arbitrary edge in cycle $C_{11}$ is as shown in Figure 2.

2.5 Theorem: Joint sum of two copies of $C_{n}$ of even order produces an E-cordial graph.

Proof: We denote the vertices of first copy of $C_{n}$ by $v_{1}, v_{2}, \ldots . v_{n}$ and second copy by $v_{1}^{\prime}, v_{2}^{\prime}, v_{3}^{\prime}, \ldots . v_{n}^{\prime}, e_{i}$ and $e_{i}^{\prime}$ where $1 \leq i \leq n$ be the corresponding edges. Join the two copies of $C_{n}$ with a new edge and let $G$ be the resultant graph. Without loss of generality we assume that the new edge be $e=v_{1} v_{1}^{\prime}$. To define $f: E(G) \rightarrow\{0,1\}$ two cases are to be considered.

Case $1: n \equiv 0(\bmod 4)$

$$
\begin{aligned}
& \begin{aligned}
f(e) & =0 ; \\
f\left(e_{i}\right) & =0 ; \text { for } i \equiv 0,1(\bmod 4), \\
& =1 ; \text { for } i \equiv 2,3(\bmod 4) \text { and } 1 \leq i \leq n
\end{aligned} \\
& \begin{aligned}
f\left(e_{i}^{\prime}\right) & =0 ; \text { for } i \equiv 0,1(\bmod 4), \\
& =1 ; \text { for } i \equiv 2,3(\bmod 4) \text { and } 1 \leq i \leq n
\end{aligned}
\end{aligned}
$$

Case $2: n \equiv 2(\bmod 4)$

$$
\begin{aligned}
& \begin{aligned}
f(e) & =1 ; \\
f\left(e_{i}\right) & =0 ; \text { for } i \equiv 0,1(\bmod 4), \\
& =1 ; \text { for } i \equiv 2,3(\bmod 4) \text { and } 1 \leq i \leq n
\end{aligned} \\
& \begin{aligned}
f\left(e_{i}^{\prime}\right) & =0 ; \text { for } i \equiv 0,1(\bmod 4), \\
& =1 ; \text { for } i \equiv 2,3(\bmod 4) \text { and } 1 \leq i \leq n
\end{aligned}
\end{aligned}
$$

In view of the above defined labeling pattern $f$ satisfies the conditions for E-cordial labeling as shown in Table 3 . That is, the joint sum of two copies of even cycle $C_{n}$ is E-cordial.

2.6 Illustration: E-cordial labeling of joint sum of two copies of cycle $C_{10}$ is shown in Figure 3.

2.7 Theorem: The split graph of $C_{n}$ of even order is E-cordial.

Proof: Let $v_{1}, v_{2}, \ldots v_{n}$ be the vertices of cycle $C_{n}$ and $v_{1}^{\prime}, v_{2}^{\prime}, \ldots v_{n}^{\prime}$ be the newly added vertices where $n$ is even. Let $G$ be the split graph of cycle $C_{n}$ with $V(G)=\left\{v_{i}, v_{i}^{\prime} ; 1 \leq i \leq n\right\}$ and $E(G)=\left\{v_{i} v_{i+1} ; 1 \leq i \leq n-1, v_{n} v_{1}, v_{i}^{\prime} v_{i+1} ; 1 \leq i \leq\right.$ $\left.n-1, v_{n}^{\prime} v_{1}, v_{i} v_{i+1}^{\prime} ; 1 \leq i \leq n-1, v_{n} v_{1}^{\prime}\right\}$. To define $f: E(G) \rightarrow\{0,1\}$ two cases are to be considered.

Case $1: n \equiv 0(\bmod 4)$

For $1 \leq i \leq n-1$ 


$$
\begin{aligned}
& f\left(v_{i} v_{i+1}\right)=0 \text {; if } i \text { is odd } \\
& =1 \text {; if } i \text { is even. }
\end{aligned}
$$

For $1 \leq i \leq n-1$

$$
\begin{aligned}
f\left(v_{i} v_{i+1}^{\prime}\right)=0 ; \text { if } i \equiv 1,2(\bmod 4) \\
=1 ; \text { if } i \equiv 0,3(\bmod 4)
\end{aligned}
$$

$f\left(v_{n} v_{1}^{\prime}\right)=1$

For $1 \leq i \leq n-1$

$$
\begin{aligned}
f\left(v_{i}^{\prime} v_{i+1}\right)=1 ; \text { if } i \equiv 1,2(\bmod 4) \\
=0 ; \text { if } i \equiv 0,3(\bmod 4)
\end{aligned}
$$

$f\left(v_{n}^{\prime} v_{1}\right)=0$

Case $2: n \equiv 2(\bmod 4)$

For $1 \leq i \leq n-1$

$$
\begin{aligned}
f\left(v_{i} v_{i+1}\right) & =0 ; \text { if } i \text { is odd } \\
& =1 \text {; if } i \text { is even. }
\end{aligned}
$$

$f\left(v_{n} v_{1}\right)=0$

For $1 \leq i \leq n-1$

$$
\begin{aligned}
f\left(v_{i} v_{i+1}^{\prime}\right)=0 ; \text { if } i \equiv 1,2(\bmod 4) \\
=1 ; \text { if } i \equiv 0,3(\bmod 4)
\end{aligned}
$$

$f\left(v_{n} v_{1}^{\prime}\right)=1$

For $1 \leq i \leq n-1$

$$
\begin{aligned}
f\left(v_{i}^{\prime} v_{i+1}\right)=1 ; \text { if } i \equiv 1,2(\bmod 4) \\
=0 ; \text { if } i \equiv 0,3(\bmod 4)
\end{aligned}
$$

$f\left(v_{n}^{\prime} v_{1}\right)=1$

In view of the above defined labeling pattern $f$ satisfies the conditions for E-cordial labeling as shown in Table 5. That is, the split graph of even cycle $C_{n}$ is E-cordial.

Illustration 2.8: Figure 4 shows the E-cordial labeling of split graph of cycle $C_{8}$.

2.9 Theorem: $D_{2}\left(P_{n}\right)$ is E-cordial for even $n$.

Proof:Let $P_{n}^{\prime} P_{n}^{\prime \prime}$ be two copies of path $P_{n}$. We denote the vertices of first copy of $P_{n}$ by $v_{1}^{\prime}, v_{2}^{\prime}, \ldots . v_{n}^{\prime}$ and second copy by $v_{1}^{\prime \prime}, v_{2}^{\prime \prime}, \ldots . v_{n}^{\prime \prime}$. Let $G$ be $D_{2}\left(P_{n}\right)$ with $|v(G)|=2 n$ and $|E(G)|=4 n-4$. To define $f: E(G) \rightarrow\{0,1\}$ two cases are to be considered.

Case $1: n \equiv 0(\bmod 4)$

For $1 \leq i \leq n-1$

$$
\begin{aligned}
f\left(v_{i}^{\prime} v_{i+1}^{\prime}\right) & =1 ; \text { if } i \neq 3 j \\
& =0 ; \text { if } i=3 j, j=1,2, \ldots\left\lfloor\frac{n}{3}\right\rfloor
\end{aligned}
$$

For $1 \leq i \leq n-1$

$$
\begin{aligned}
f\left(v_{i}^{\prime \prime} v_{i+1}^{\prime \prime}\right) & =0 ; \text { if } i \neq 3 j \\
& =1 \text {; if } i=3 j, j=1,2, \ldots\left\lfloor\frac{n}{3}\right\rfloor
\end{aligned}
$$

For $1 \leq i \leq n-2$

$$
\begin{aligned}
f\left(v_{i}^{\prime \prime} v_{i+1}^{\prime}\right) & =1 ; \text { if } i \text { is odd } \\
& =0 \text {; if } i \text { is even }
\end{aligned}
$$




$$
\begin{aligned}
& f\left(v_{n-1}^{\prime \prime} v_{n}^{\prime}\right)=0 \\
& \begin{aligned}
& \text { For } 1 \leq i \leq n-1 \\
& f\left(v_{i}^{\prime} v_{i+1}^{\prime \prime}\right)=1 ; \text { if } i \text { is odd } \\
&=0 \text {; if } i \text { is even }
\end{aligned}
\end{aligned}
$$

Case $2: n \equiv 2(\bmod 4)$

For $1 \leq i \leq n-1$

$$
\begin{aligned}
f\left(v_{i}^{\prime} v_{i+1}^{\prime}\right) & =1 ; \text { if } i \equiv 1,2(\bmod 4) \\
& =0 ; \text { if } i \equiv 0,3(\bmod 4)
\end{aligned}
$$

For $1 \leq i \leq n-1$

$$
\begin{aligned}
f\left(v_{i}^{\prime \prime} v_{i+1}^{\prime \prime}\right) & =0 ; \text { if } i \equiv 1,2(\bmod 4) \\
& =1 ; \text { if } i \equiv 0,3(\bmod 4)
\end{aligned}
$$

For $1 \leq i \leq n-2$

$$
\begin{aligned}
f\left(v_{i}^{\prime} v_{i+1}^{\prime \prime}\right) & =1 ; \text { if } i \text { is odd } \\
& =0 \text {; if } i \text { is even }
\end{aligned}
$$

$f\left(v_{n-1}^{\prime} v_{n}^{\prime \prime}\right)=0$

For $1 \leq i \leq n-1$

$f\left(v_{i}^{\prime \prime} v_{i+1}^{\prime}\right)=1$; if $i$ is odd

$$
=0 \text {; if } i \text { is even }
$$

In view of the above defined labeling pattern $f$ satisfies the conditions for E-cordial labeling as shown in Table 5. That is, $D_{2}\left(P_{n}\right)$ is E-cordial for even $n$.

Illustration 2.10: Figure 5 shows the E-cordial labeling of $D_{2}\left(P_{6}\right)$.

\section{Concluding Remarks}

Here we contribute five new families of E-cordial graphs generated by different graph operations. To investigate similar results for other graph families and in the context of different graph labeling techniques is an open area of research.

\section{Acknowledgment}

The authors are highly thankful to the anonymous referees for constructive suggestions and comments.

\section{References}

Bloom, G. S. and Golomb, S. W. (1977). Applications of numbered undirected graphs. Proceedings of IEEE, 65(4), 562-570. http://dx.doi.org/10.1109/PROC.1977.10517

Cahit, I. (1987). Cordial Graphs: A weaker version of graceful and harmonious graphs. Ars Combinatoria, 23, 201-207.

Devraj, J. (2004). On edge-cordial graphs.Graph Theory Notes of New York, XLVII, 14-18.

Gallian, J. A. (2010). A dynamic survey of graph labeling. The Electronics Journal of Combinatorics, 17, \#DS 6.

Harary, F. (1972). Graph Theory. Addison Wesley, Reading, Massachusetts.

Rosa, A. (1966, 1967). On certain valuation of the vertices of a graph. Theory of graphs, International Symposium, Rome, New York and Dunod Paris ,349-355.

Yilmaz, R. and Cahit, I. (1997). E-cordial graphs. Ars Combin, 46, 251-266.

Table 1.

\begin{tabular}{|l|l|l|}
\hline$n$ & vertex condition & edge condition \\
\hline \hline$n \cong 0(\bmod 4)$ & $v_{f}(0)=v_{f}(1)+1=\frac{n+2}{2}$ & $e_{f}(0)=e_{f}(1)=\frac{n+2}{2}$ \\
\hline$n \cong 2(\bmod 4)$ & $v_{f}(1)=v_{f}(0)+1=\frac{n+2}{2}$ & $e_{f}(0)=e_{f}(1)=\frac{n+2}{2}$ \\
\hline$n \cong 3(\bmod 4)$ & $v_{f}(1)=v_{f}(0)=\frac{n+1}{2}$ & $e_{f}(1)=e_{f}(0)+1=\frac{n+3}{2}$ \\
\hline
\end{tabular}


Table 2.

\begin{tabular}{|l|l|l|}
\hline$n$ & vertex condition & edge condition \\
\hline \hline$n \cong 1(\bmod 4)$ & $v_{f}(1)=v_{f}(0)+1=\frac{n+3}{2}$ & $e_{f}(0)=e_{f}(1)=\frac{n+3}{2}$ \\
\hline$n \cong 2(\bmod 4)$ & $v_{f}(1)=v_{f}(0)=\frac{n+2}{2}$ & $e_{f}(1)=e_{f}(0)+1=\frac{n+4}{2}$ \\
\hline$n \cong 3(\bmod 4)$ & $v_{f}(0)=v_{f}(1)+1=\frac{n+3}{2}$ & $e_{f}(0)=e_{f}(1)=\frac{n+3}{2}$ \\
\hline
\end{tabular}

Table 3.

\begin{tabular}{|l||l|l|}
\hline$n$ & Vertex Condition & Edge Condition \\
\hline \hline$n \cong 0(\bmod 4)$ & $v_{f}(0)=v_{f}(1)=n$ & $e_{f}(0)=e_{f}(1)+1=n+1$ \\
\hline$n \cong 2(\bmod 4)$ & $v_{f}(0)=v_{f}(1)=n$ & $e_{f}(1)=e_{f}(0)+1=n+1$ \\
\hline
\end{tabular}

Table 4.

\begin{tabular}{|l|l|l|}
\hline$n$ & Vertex Condition & Edge Condition \\
\hline \hline$n \cong 0,2(\bmod 4)$ & $v_{f}(0)=v_{f}(1)=n$ & $e_{f}(0)=e_{f}(1)=\frac{3 n}{2}$ \\
\hline
\end{tabular}

Table 5.

\begin{tabular}{|l|l|l|}
\hline$n$ & Vertex Condition & Edge Condition \\
\hline \hline$n \cong 0,2(\bmod 4)$ & $v_{f}(0)=v_{f}(1)=n$ & $e_{f}(0)=e_{f}(1)=2 n-2$ \\
\hline
\end{tabular}

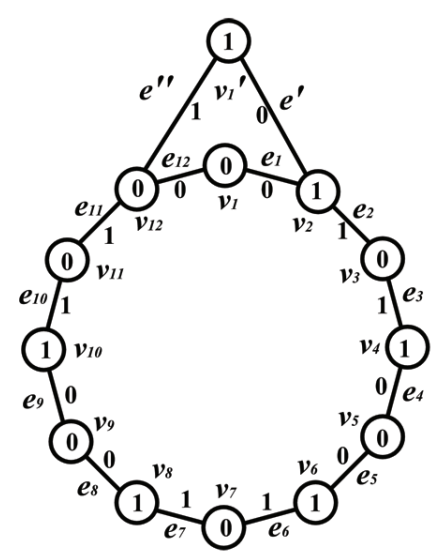

Figure 1. E-cordial labeling of the graph obtained by duplication of vertex $v_{1}$ in $C_{12}$

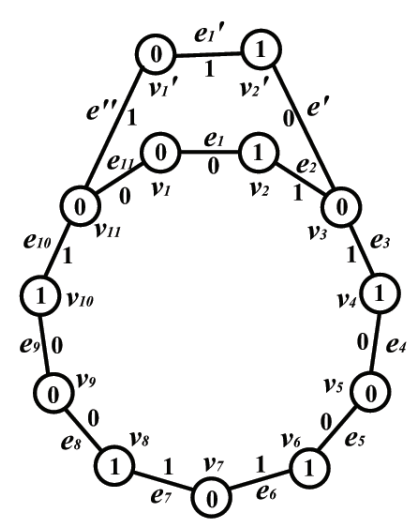

Figure 2. E-cordial labeling of the graph obtained by duplication of the edge $v_{1} v_{2}$ in $C_{11}$ 


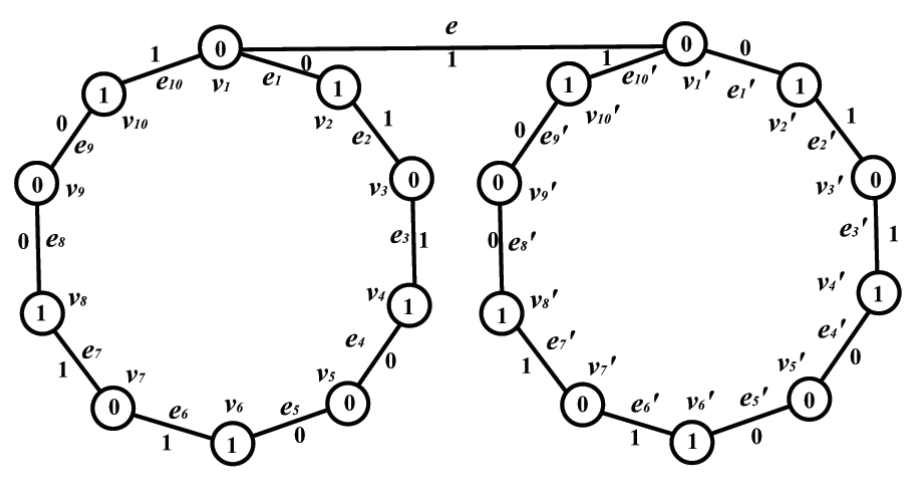

Figure 3. E-cordial labeling of Joint sum of two copies of $C_{10}$

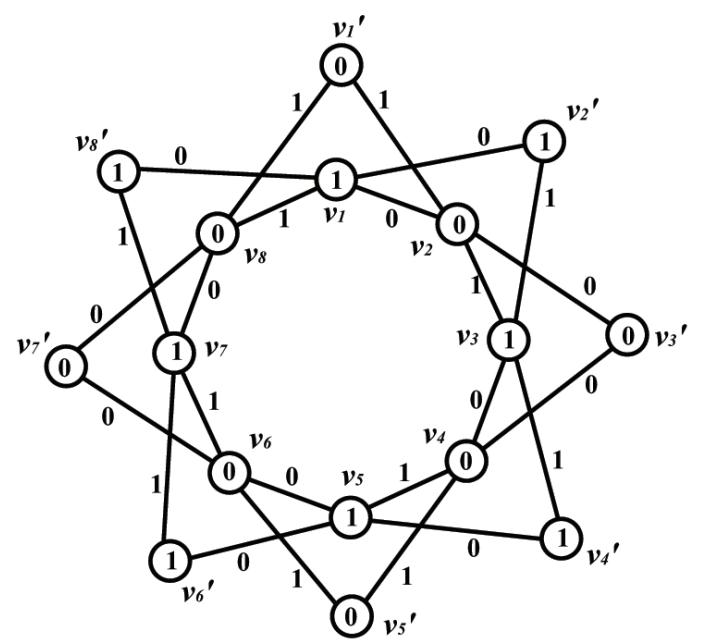

Figure 4. E-cordial labeling of split graph of cycle $C_{8}$

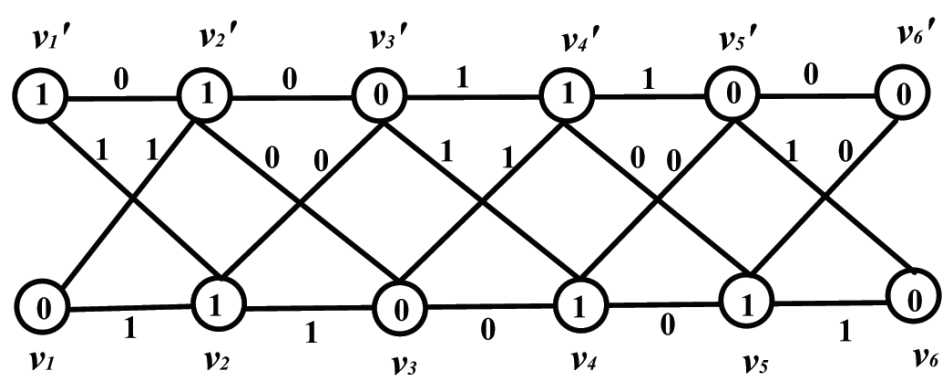

Figure 5. E-cordial labeling of $D_{2}\left(P_{6}\right)$ 